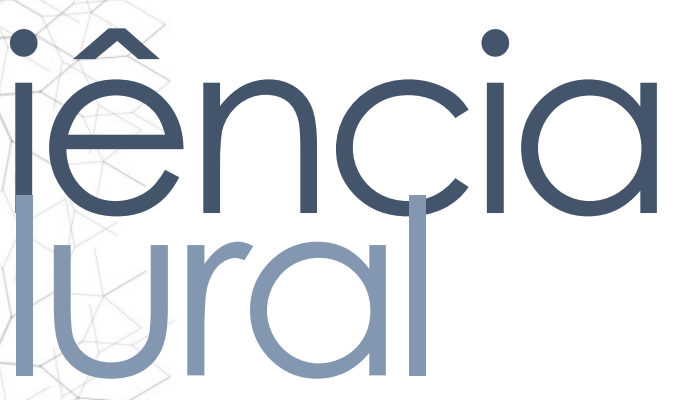

\title{
CONDIÇÕES DE SAÚDE E ESTADO NUTRICIONAL DE AGENTES COMUNITÁRIOS DE SAÚDE NO INTERIOR DO NORDESTE BRASILEIRO
}

Health conditions and nutritional status of community health agents in the interior of the Brazilian Northeast

Condiciones de salud y condiciones nutricionales de agentes de salud comunitarios en el noreste de Brasil

Amanda Almeida Gomes Dantas • Universidade Federal do Rio Grande do NorteUFRN • Nutricionista • Mestra em Saúde Coletiva • E-mail: almmeidaamanda@gmail.com

Nayara Priscila Dantas de Oliveira - Universidade Federal do Rio Grande do Norte-UFRN • Fisioterapeuta • Mestra em Saúde Coletiva $\bullet$ E-mail: nayoliveira.fisio@gmail.com

Mercês de Fátima dos Santos Silva • Professora da Universidade Federal do Rio Grande do Norte-UFRN • Cientista Social • Doutora em Saúde Coletiva • E-mail: merces.santos30@gmail.com

Diego de Sousa Dantas • Professor da Universidade Federal de Pernambuco • Fisioterapeuta • Doutor em Biotecnologia • E-mail: diegodantas1@gmail.com

Autora responsável pela correspondência:

Amanda Almeida Gomes Dantas E-mail: almmeidaamanda@gmail.com 


\section{RESUMO}

Introdução: A saúde do trabalhador é um campo de relevância, mas que ainda está sendo consolidado no âmbito do sistema único de saúde (SUS). Partindo dessa compreensão, esse estudo teve como objetivo avaliar o estado nutricional e as condições de saúde dos agentes comunitários de saúde do município de Currais Novos-RN. Métodos: Trata-se de um estudo transversal, realizado com 80 indivíduos de ambos os sexos. Condições sociais e de saúde, estilo de vida e estado nutricional foram investigados. A análise bivariada foi conduzida através do teste Qui-quadrado, para analisar a associação entre o estado nutricional e as variáveis independentes. Considerou-se o nível de significância estatística de $p \leq 0,05$. Resultados: A maioria dos participantes encontravam-se na faixa etária de 41 a 58 anos (52, 5\%), possuía renda maior que 1 salário mínimo (77,5\%) e encontrava-se com Sobrepeso/Obesidade $(63,8 \%)$. Apenas as variáveis antropométricas percentual de gordura $(p<0,02)$, relação cintura/quadril $(p<0,02)$ e satisfação com peso $(p<0,01)$ se associaram ao estado nutricional. Conclusão: $O$ presente estudo verificou que a maioria dos indivíduos estudados apresenta sobrepeso/obesidade, que os dados referentes a condições sociais e de saúde, como também estilo de vida não se associaram ao desfecho que foi analisado. As variáveis antropométricas apresentaram diferença significativa na análise bivariada, demonstrando que estas exercem influência no estado nutricional.

Palavras-chaves: Agente Comunitário de Saúde; Saúde do Trabalhador; Condições de Saúde; Estado Nutricional.

\section{ABSTRACT}

Introduction: Occupational health is a relevant field, but it is still being consolidated within the scope of the single health system (SUS). From this understanding, this study aimed to evaluate the nutritional status and health conditions of community health agents in the city of Currais Novos-RN. Methods: This is a cross-sectional study, conducted with 80 individuals of both sexes.Social and health conditions, lifestyle and nutritional status were investigated. Bivariate analysis was conducted using the Chisquare test to analyze the association between nutritional status and independent variables. The level of statistical significance of $p \leq 0,05$ was considered. Results: Most participants were between 41 and 58 years old (52,5\%), had an income higher than 1 minimum wage $(77,5 \%)$ and were overweight / obese $(63,8 \%)$. Only the anthropometric variables percentage of fat $(\mathrm{p}<0,02)$, waist / hip ratio $(\mathrm{p}<0,02)$ and weight satisfaction $(p<0,01)$ were associated with nutritional status. Conclusion: The present study found that most of the individuals studied were overweight / obese, that data regarding social and health conditions, as well as lifestyle, were not associated with the outcome that was analyzed. The anthropometric variables showed a significant difference in the bivariate analysis, demonstrating that they influence the nutritional status. 
Keywords: Community Health; Agent Worker's Health; Health Conditions; Nutritional Status.

\section{RESUMEN}

Introducción: La salud ocupacional es un campo relevante, pero aún se está consolidando dentro del alcance del sistema único de salud (SUS). En base a esta comprensión, este estudio tuvo como objetivo evaluar el estado nutricional y las condiciones de salud de los agentes de salud comunitarios en la ciudad de Currais Novos-RN. Métodos: Este es un estudio transversal realizado con 80 individuos de ambos sexos. Se investigaron las condiciones sociales y de salud, el estilo de vida y el estado nutricional. El análisis bivariado se realizó mediante la prueba de Chi-cuadrado para analizar la asociación entre el estado nutricional y las variables independientes. Se consideró el nivel de significación estadística de $\mathrm{p} \leq 0.05$. Resultados: La mayoría de los participantes tenían entre 41 y 58 años (52.5\%), tenían un ingreso superior a 1 salario mínimo (77.5\%) y tenían sobrepeso / obesidad (63.8\%). Solo las variables antropométricas porcentaje de grasa $(p<0.02)$, relación cintura / cadera $(p<0.02)$ y satisfacción con el peso $(p<0.01)$ se asociaron con el estado nutricional. Solo las variables antropométricas porcentaje de grasa $(\mathrm{p}<0.02)$, relación cintura / cadera ( $\mathrm{p}$ $<0.02)$ y satisfacción con el peso $(\mathrm{p}<0.01)$ se asociaron con el estado nutricional. Conclusión: El presente estudio encontró que la mayoría de los individuos estudiados tenían sobrepeso / obesidad, y los datos sobre las condiciones sociales y de salud, así como el estilo de vida, no se asociaron con el resultado que se analizó. Las variables antropométricas mostraron una diferencia significativa en el análisis bivariado, lo que demuestra que influyen en el estado nutricional.

Palabras clave: Agente de salud comunitaria; Salud ocupacional; Condiciones de salud; Estado nutricional. 


\section{Introdução}

A saúde do trabalhador compreende um campo de práticas e de conhecimentos táticos interdisciplinares - técnicos, sociais, políticos, humanos, multiprofissionais e interinstitucionais, direcionados para avaliar e intervir nas relações de trabalho que geram doenças e agravos. Seus marcos referenciais são da Saúde Coletiva, e procura, assim, constituir causas de agravos à sua saúde, reconhecer seus determinantes, aferir riscos, dar a conhecer os modos de prevenção e promover à saúde ${ }^{1}$.

O movimento de cuidado da saúde do trabalhador, é inerente do crescente adoecimento dessa parcela da população, no qual estão inseridos aqueles que cuidam da saúde dos indivíduos. Estes trabalhadores que se empenham em direcionar seu olhar para o cuidado do outro, por vezes, esquecem de atentar para sua própria saúde, como também para as condições do seu ambiente de trabalho ${ }^{2}$.

Inseridos nesse contexto, estão os Agentes Comunitários de Saúde (ACS), que são profissionais que compõem a equipe da Estratégia de Saúde da Família (ESF), que foi implementa em 1994 em todo o país, objetivando a reorientação do modelo assistencial da saúde, baseada e orientada pelos princípios e diretrizes do Sistema Único de Saúde (SUS) ${ }^{3}$.

Na atenção básica em saúde, o ACS é um elemento-chave do sistema, cabendo a esses trabalhadores realizar atividades de prevenção de doenças e promoção de saúde, por meio de ações educativas em saúde, realizadas em domicílios ou junto à coletividade ${ }^{4}$.

Esses trabalhadores apresentam uma sobrecarga de trabalho e de funções ${ }^{4}$ o que pode interferir na ingestão de uma alimentação adequada e na prática de exercício físico, podendo afetar o estado de saúde, incidindo no desempenho e a produtividade do serviço prestado por estes 5 .

Nesse contexto, destaca-se a importância de se investigar as condições de saúde o estado nutricional desses profissionais, visto que existe uma lacuna de nhecimento no meio científico sobre esse desfecho. Assim, esse estudo tem como etivo avaliar as condições de saúde e o estado nutricional dos agentes comunitários saúde do município de Currais Novos-RN. 


\section{Metodologia}

Trata-se de um estudo transversal, realizado no município de Currais NovosRN, no período de agosto a outubro de 2017. O protocolo de pesquisa foi submetido e aprovado pelo Comitê local de Ética em Pesquisa sob o número CAAE: 68438317.6.0000.5568. O estudo foi conduzido em consonância com os termos da Resolução 466/12 do Conselho Nacional de Saúde.

Foi definida uma amostragem não-probabilística, definida por acessibilidade aos sujeitos, e dos 110 funcionários registrados como ACS na secretaria de saúde, foram arrolados no estudo 80 indivíduos. Foram incluídos os profissionais que estavam desempenhando sua função, como também, os que se encontravam na unidade básica de saúde no dia da realização da coleta de dados, e que aceitaram participar da pesquisa por meio da assinatura do termo de consentimento livre e esclarecido. Foram excluídos do estudo, aqueles que estavam no serviço a menos de um ano.

A coleta de dados ocorreu nas 17 unidades básicas de saúde, localizadas na cidade de Currais Novos-RN. A mesma se deu, através da aplicação do questionário estruturado que permitiu a obtenção dos dados relativos as condições de saúde e vida desses trabalhadores, divido em cinco tópicos, que foram as seguintes: Identificação dos trabalhadores; antropometria; estilo de vida; aspectos de saúde e condições sociais. Este questionário foi elaborado com base na Pesquisa Nacional de Saúde, realizada por o Instituto Brasileiro de Geografia e Estatística ${ }^{6}$ e adaptado ao público alvo desse estudo.

Além disso, foi realizada a aferição das medidas antropométricas de peso corporal, estatura, circunferência abdominal, dobras cutâneas tricipital, abdominal, supra- ilíaca e subescapular. Com estas informações foi possível realizar cálculo do indice de Massa Corporal (IMC), do percentual de gordura corporal, e analisar o Risco

Desenvolvimento de Doenças Cardiometabólicas.

Para aferição do peso corporal foi utilizada balança digital Ultra Sim, da marca iso Care, modelo W910 com capacidade máxima de 180 Kg e graduação de 100 g. Já tatura foi mensurada apenas uma vez, e foi realizada por meio de fita métrica não tica do tipo Fiberglass, fixada na parede sem rodapé. A partir das medidas de peso 
e estatura foi calculado o IMC, que em seguida foi classificado de acordo com os critérios preconizados pela Organização Mundial de Saúde $(\mathrm{OMS})^{7}$, que são: baixo peso, adequado, sobrepeso e obesidade.

A medida de circunferência abdominal, foi medida por meio de fita métrica não elástica, e a mesma é aferida na cicatriz umbilical. Por meio dessa, foi possível avaliar o risco de desenvolvimento de doenças cardiometabólicas com os seguintes pontos de corte: $>80,0 \mathrm{~cm}$ para mulheres e $>90,0 \mathrm{~cm}$ para homens, de acordo com as recomendações das Diretrizes Brasileiras de Obesidade ${ }^{8}$.

O percentual de gordura foi calculado através da aferição das dobras cutâneas tricipital, abdominal, supra-ilíaca e subescapular. Para a aferição das dobras foi utilizado um adipômetro da marca Opus max, com faixa de medição de 0 a 92 mm. Foram realizadas duas medidas em cada local, e obtida a média, de acordo com o que é preconizado 9 .

Para análise do percentual de gordura, que diferem segundo o sexo, foram utilizados como critérios para classificação segundo Faulkner, os seguintes: baixo, adequado, moderadamente acima, excesso e obesidade. Para aquelas pessoas que apresentavam obesidade, o que impossibilitava a aferição das dobras, o percentual de gordura foi calculado através do Protocolo de Weltman, que utiliza a medida da circunferência abdominal ${ }^{9}$.

Esse estudo tem como variável dependente, estado nutricional, determinado a partir do IMC, cujo escore final é categorizado ordinalmente em quatro categorias, que são as seguintes: baixo peso, adequado, sobrepeso e obesidade. Mas, para analise bivariada, o mesmo foi recategorizado em duas categorias, a) Estado nutricional adequado e b) sobrepeso/obesidade. As variáveis independentes do estudo são informações relacionadas a aspectos socioeconômicos, estilo de vida, acesso aos serviços de saúde, aspectos de saúde, condições sociais e aspectos antropométricos.

Para as variáveis quantitativas, a adequação dos dados à distribuição normal avaliada levando em consideração alguns critérios estabelecidos: Valores mínimo náximo estarem contidos no intervalo correspondente ao cálculo da média \pm três vios padrões. 


\section{ciência
piural}

Os dados foram tabulados e analisados através do software Statistical Package for the Social Sciences (SPSS) versão 20.0. Inicialmente, os resultados foram apresentados por meio de estatística descritiva, mediante o uso de tabelas com valores absolutos e relativos dos dados. Em seguida foi realizada a análise bivariada, utilizando-se o teste de Qui-quadrado, sendo verificada a magnitude da associação através da RP para cada uma das variáveis independentes categóricas nominais em relação a variável dependente. Considerou-se o nível de significância estatística de p $\leq 0,05$.

\section{Resultados}

Foram incluídos no estudo 80 trabalhadores. Destes, $82,5 \%$ foram do sexo feminino, e 17,5\% do masculino. A maioria das participantes encontravam-se na faixa etária de 24 a 40 anos (52,5\%), tinham companheiros (52,5\%), e possuíam ensino médio ou superior $(96,2 \%)$. No que diz respeito à renda mensal a grande maioria das participantes possui renda familiar mensal maior que um salário mínimo $(77,5 \%)$ (Tabela 1).

Tabela 1- Análise Bivariada da caracterização da população do estudo, aspectos sociais e de saúde, estilo de vida e aspectos antropométricos dos ACS. Currais Novos/RN. Brasil, 2017.

\section{Estado Nutricional}

$$
\begin{gathered}
\text { Geral }(\mathrm{n}=80) \quad \begin{array}{c}
\text { Sobrepeso/Obesida } \\
\text { de } \\
(\mathrm{n}=51)
\end{array} \\
\hline
\end{gathered}
$$$$
\text { Adequado }
$$$$
(n=29)
$$

n $\quad \% \quad$ n

$\%$

$\mathbf{n}$

$\% \quad$ p valor

Faixa Etária

41 a 58 anos

24 a 40 anos

\section{8}

42

$\begin{array}{ll}47,5 & 27,0 \\ 52,5 & 24,0\end{array}$

66

14

$$
82,5
$$

42,0

17,5

9,0

53,0

47,0

15,0

51,7

0,92

Sexo

Masculino

Escolaridade

Ensino Me. Inc.

Ensino Me. Comp./ Superior

Renda

Até 1 salário mínimo

$>1$ salário mínimo

Estado Civil

$\begin{array}{ccccccc}3 & 3,8 & 2,0 & 4,0 & 1,0 & 3,5 & 1,00 \\ 77 & 96,2 & 49,0 & 96,0 & 28,0 & 96,5 & \\ & & & & & & \\ 18 & 22,5 & 11,0 & 21,6 & 7,0 & 24,1 & 0,80 \\ 62 & 77,5 & 40,0 & 78,4 & 22,0 & 75,9 & \end{array}$


Sem companheiro

Com companheiro

Comodo na Residência

4 a 5

6 a 10

Esgotamento Sanitário

Rede Geral

Outro

Diagnóstico Hipertensão

Sim

Não

Diagnóstico Colesterol

Sim

Não

Diagnóstico Diabetes

Sim

Não

Atividade Física

Sim

Não

Consumo de Álcool

Sim

Não

Classificação Percentual de

Gordura

Não Adequado

Adequado

Classificação Circunferência

Abdominal

Risco

Não Risco

Satisfeito com o Peso

Insatisfeito

Satisfeito

$$
38
$$

42

47,5

11,0

40,0

25

55

31,3

14,0

68,8

37,0

51

29

51

63,7

30,0

$36,3 \quad 21,0$

$17 \quad 21,3 \quad 13,0$

$63 \quad 78,7 \quad 38,0$

$22 \quad 27,5 \quad 14,0$

$58 \quad 72,5 \quad 37,0$

$\begin{array}{lll}5 & 6,2 & 3,0\end{array}$

$75 \quad 93,8 \quad 48,0$

$32 \quad 40,0$

23,0

48

60,0

28,0

$44 \quad 55,0$

28,0

36

45,0

23,0

38

42

47,5

52,5

31,0

20,0

60,9

39,1

7,0

22,0

$6,0 \quad 20,7$

0,27

74,5

$23,0 \quad 79,3$

27,5

72,5

$9,0 \quad 31,0$

0,91

$20,0 \quad 69,0$

\section{5,9}

94,1

2,0

6,9

1,01

$27,0 \quad 93,1$

45,1

54,9

9,0

31,0

0,21

54,9

45,1

16,0

55,2

$13,0 \quad 44,8$

0,98

( 


\section{Discussão}

Ao analisar o estado nutricional e as condições de saúde dos ACS, residentes em município do interior do nordeste brasileiro, verificou-se que 63,8\% encontra-se com sobrepeso/obesidade, e que este estado nutricional esteve associado apenas ao estado civil e os aspectos antropométricos. Demais variáveis investigadas, como idade, atividade física, escolaridade e aspectos de saúde não se associaram a essa condição.

Dos participantes desse estudo $82,5 \%$ foram do sexo feminino. A predominância de mulheres na profissão foi demonstrada em outros estudos com ACS8,9. Alguns autores acabam referindo que isso acontece devido ao fato da população mostrar resistência ao agente do sexo masculino ${ }^{10}$, e por outro lado, as mulheres são maioria também nessa profissão em virtude do papel de cuidadora que exercem em nossa sociedade ${ }^{11}$.

Nessa pesquisa o estado civil se associou ao estado nutricional. Uma plausível explicação para esse achado seria que, após a união o indivíduo deixa de se cuidar no que diz respeito a aparência física, e isso colabora para o ganho de peso corporal.

Não foi observada a associação do estado nutricional com a prática de atividade física. Talvez isso se deva ao fato do cansaço oriundo da grande demanda física exigida pelas visitas diárias realizadas por os ACS. A literatura evidencia os inúmeros benéficos que a pratica de atividade física proporciona como, perda de peso com diminuição da gordura corporal, aumento de massa magra e da captação de insulina, o que proporciona uma melhora no desenvolvimento da capacidade laboral de trabalhadores ${ }^{12}$.

No que diz relação as condições de saúde, nessa pesquisa observou-se que a grande maioria dos ACS não receberam diagnóstico de doenças crônicas não transmissíveis (DCNT). Esse fato pode ser explicado, porque (52,2\%) dos participantes encontra-se na faixa etária de 24 a 40 anos, onde a prevalência dessas patologias é menor. Ultimamente, as DCNT compõem o problema de saúde de maior amplitude e espondem por mais de $70 \%$ dos motivos de mortes no Brasil. As doenças rdiovasculares, câncer, diabetes, enfermidades respiratórias crônicas e doenças uropsiquiátricas, principais DCNT, têm sido responsáveis por um número alto de 
mortes e perda de qualidade de vida, causando incapacidades e alto grau de limitação das pessoas doentes em suas atividades de trabalho e de lazer ${ }^{13}$.

Com relação ao estado nutricional desses trabalhadores, de acordo com os dados antropométricos, viu-se que mais da metade dos participantes $(63,8 \%)$ encontram-se com sobrepeso/obesidade. De acordo com a literatura o excesso de peso é consequente da relação complexa entres inúmeros fatores, entre os quais podem ser inclusas as características do trabalho. As condições adversas de trabalho, tais como extensas jornadas, demandas excessivas e exposição a ambientes hostis, podem contribuir para a prevalência de obesidade na população trabalhadora, explicando os resultados encontrados nesse trabalho ${ }^{14}$. Vale salientar que o excesso de peso corporal tem sido considerado uma epidemia, devido ao aumento de sua prevalência em todas as faixas etárias ${ }^{15}$.

A circunferência abdominal se associou ao estado nutricional. Mais da metade dos participantes desse estudo apresentam risco, segundo essa medida para o desenvolvimento de doenças cardiometabólicas, segundo as Diretrizes Brasileiras de Obesidade $^{8}$. O mesmo foi encontrado no estudo realizado por Freitas ${ }^{16}$, no qual 46,3\% apresentavam risco de desenvolvimento dessas patologias. A obesidade abdominal merece um olhar diferenciado, uma vez que essa estar associada a um maior risco para a saúde pela distribuição de gordura mais periférica, podendo futuramente levar ao desenvolvimento de DCNT17.

Os ACS foram indagados sobre o que achavam do seu peso e $67,5 \%$ afirmaram que julgavam estar acima do desejado, achado que leva ao entendimento de que todos os indivíduos com sobrepeso/obesidade, deste estudo, têm a consciência que estão acima do peso, fator importante para a tomada de decisão de adoção de hábitos de vida mais saudáveis.

Como limitações do estudo, pontuamos o tamanho amostral limitado para associações estatísticas, justificado pela dificuldade de acesso à amostra, o que pode ter interferido na análise com relação a significância dos dados relacionados as condições de vida e saúde. Como também, destaca-se o desenho transversal que limita a interpretação dos dados a associações, passíveis de causalidade reversa, e ainda 
impedem uma melhor compreensão do fenômeno pela incapacidade de se estabelecer causalidade ${ }^{18}$.

\section{Conclusões}

A maioria dos ACS encontra-se com sobrepeso/obesidade, e esse estado nutricional se associou ao estado civil e aspectos antropométricos. Fatores sociodemográficos, aspectos de saúde e hábitos de vida como atividade física, não apresentaram associação com essa condição.

Os resultados encontrados podem ser úteis como sinais de alerta para que esses profissionais de saúde, que apresentaram excesso de peso, percentual de gordura corporal elevado, como também risco para doenças cardiometabólicas, cuidem de sua saúde para assim prevenirem o desenvolvimento de doenças que estão anexas as condições apresentadas, dessa forma contribuindo para manutenção de uma condição de saúde adequada.

\section{Referências}

1. Mendes R; Dias EC. Da medicina do trabalho à saúde do trabalhador. Revista de Saúde Pública, São Paulo, 1991;25(5): 341-342.

2. Gomes MLP; Silva JCB; Batista EC. Escutando quem cuida: quando o cuidado afeta a saúde do cuidador em saúde mental. Rev. Psicol. Saúde, 2018;10(1): 03-07.

3. Ministério da Educação (BR). Proposta de formação de agentes comunitário de saúde: habilitação funcional técnica. Brasília, 2004.

4. Garcia ACP et al. Agente comunitário de saúde no espírito santo: do perfil às atividades desenvolvidas. Trab. educ. saúde. 2017; 15(1):283-300.

5. Matos $\mathrm{CH}$; Proença RPC. Condições de trabalho e estado nutricional de operadores do setor de alimentação coletiva: um estudo de caso. Rev. Nutr,2003;16(4): 493-502.

6. Instituto Brasileiro de Geografia e Estatística. Pesquisa Nacional de Saúde. [Site da Internet] 2013. [acessado agosto 2017] Disponível em: de http://www.ibge.gov.br/home/presidencia/noticias/imprensa/ppts/0000001 410010811201301571283436.pdf.

World health organization. Physical status: the use and interpretation of anthropometric. Geneva, 2006.

Diretrizes Brasileiras de Obesidade. Associação Brasileira para o Estudo da Obesidade e da Síndrome Metabólica. São Paulo, 4, 2016. 
9. Duarte ACG. Avaliação Nutricional: aspectos clínicos e laboratoriais. São Paulo: Atheneu, 2007.

10. Ferraz L.; Aerts D. O cotidiano de trabalho do agente comunitário de saúde no PSF em Porto Alegre. Ciência \& Saúde Coletiva, Rio de Janeiro, 2007; 10(2):347355.

11. Freitas AC, Marcolino FF, Santos IG. Agentes comunitários de saúde da zona leste do município de São Paulo: um olhar para seu estado nutricional e consumo alimentar. Rev Brasileira em Promoção da Saúde.2008,21 (1):3-12.

12. Leão HFP, Gomes SA, Almeida AHS, Batista GR, Thasiro T, Castro PJP. Qualidade de vida e nível de atividade física de trabalhadores com diferentes tempos de serviço. Revista Brasileira de Ciências da Saúde.2011; 15(1)31-38.

13. World Health Organization. Noncommunicable diseases country profiles 2018 [Internet]. Geneva: World Health Organization; 2018 [citado Setembro de 2019]. 223 p. Disponível em: Disponível em: https://www.who.int/nmh/publications/ncd-profiles-2018/en/

14. Hidalgo KD, Mielke GI, Parra DC, Lobelo F, Simões EJ, Gomes GO, et al. Health promoting practices and personal lifestyle behaviors of Brazilian health professionals. BMC Public Health. 2016;16(1):1114.

15. Cassiano MH, Santana Luz AB, Bezerra MS, Barbosa SS, Silva HTDDA, Araújo DFDES. Correlação entre os índices antropométricos e pressão arterial de adolescentes e adultos jovens em um município do nordeste brasileiro. Revista Ciência Plural. 2019; 5(2):49-67.

16. Freitas AC, Marcolino FF, Santos IG. Agentes comunitários de saúde da zona leste do município de São Paulo: um olhar para seu estado nutricional e consumo alimentar. Rev Brasileira em Promoção da Saúde. 2008; 21 (1): 3-14.

17. Ferreira TS, Chafauzer C, Araújo Júnior FM, Silva GB. Obesidade central em jovens. Science in Health. 2012, 3(2): 61-73.

18. Fletcher RH, Fletcher SW. Epidemiologia Clínica: elementos essenciais. $4^{\mathrm{o}}$ ed. Porto Alegre: Artmed, 2006. 